probabilities were $96.8 \%$ and $91.3 \%$. In addition it was shown by the Cox-model that patients with higher CD4 and patients without ART before KS diagnosis had a significant lower mortality risk.

Conclusion A low CD4 cell count at KS diagnosis is an important predictor for mortality.

\section{P2.127 PRESENTATION OF LYMPHOGRANULOMA VENEREUM (LGV) IN 2012 IN THE REFERENCE STI UNIT OF BARCELONA}

doi:10.1136/sextrans-2013-051184.0391

'M Arando, 'M Vall, 'P Armengol, ${ }^{2} \mathrm{E}$ Caballero, 'M Barberá. 'UITS, Barcelona, Spain; ${ }^{2}$ Hospital Universitari Vall d'Hebron, Barcelona, Spain

Since 2003 LGV outbreaks have been reported in different European cities in men who have sex patient men with high-risk sexual behaviours. In the majority of cases the most common clinical presentation was a rectal syndrome described as an acute proctitis. In Barcelona during 2012 the most common clinical presentation was proctitis, but revealed an increase in cases of LGV extrarectal location.

Objective To analyse LGV cases that have been diagnosed in 2012 in the reference STI Unit (UITS) Drassanes of Barcelona.

Methods Retrospective descriptive study. Review of epidemiological data, clinical presentation, physical examination and results of mentioned cases has been conducted.

Results In 2012 fifty-two cases of LGV in UITS were diagnosed, 8 of them in an extrarectal location. All of them were male: 50 were MSM and 2 bisexual men. Co-infection with HIV was $65 \%$, decreasing to $50 \%$ in the extrarectal location. $11.5 \%$ of them had a history of previous LGV and $36.5 \%$ had a sexually transmitted infection in the previous 12 months. The most common clinical presentation was proctitis $(65 \%)$, other clinical manifestations were urethritis, inguinal syndrome and anogenital ulcers. $21 \%$ were diagnosed by contact study (half for LGV and half for other STI) and one case was diagnosed by screening in an asymptomatic patient.

Discussion Although during the last years the typical presentation of LGV has been proctitis, the extrarectal location has increased considerably in 2012. In this situation it is necessary to assess whether or not to request it in a urethral syndrome as ulcerative lesions, especially in men who have sex with men with high-risk sexual behaviours.

\section{P2.128 GENITAL PRIMARY LYMPHOGRANULOMA VENEREUM}

doi:10.1136/sextrans-2013-051184.0392

M Vall-Mayans, E Caballero. University Hospital Vall d'Hebron, Barcelona, Spain

Lymphogranuloma venereum (LGV) in Europe emerged in 2003 affecting mostly MSM. Rectal primary LGV causing proctitis has been by far de most common clinical manifestation. In Barcelona, with a cumulative number of around 200 cases up to 2012, some cases have appeared with extrarectal manifestations since 2008. We report two cases in MSM with genital primary LGV mimicking primary syphilis.

Case 1. A 29-year-old HIV-positive man presented with a genital chancre and inguinal lymphadenopathy of 10 days' duration. He reported 5 casual partners the last 3 months. Although dark-field exam was negative the patient was treated with benzathine penicillin G 2.4 million units. He was seen 4 days later without improvement, with buboes and genital edoema. A swab from the ulcer was positive for LGV and negative for syphilis and chancroid by PCR. A diagnosis of bubonulus was made, and the patient started doxycycline $100 \mathrm{mg} / 12 \mathrm{~h}$ for 21 days. He improved persisting a small residual soft mass on the shaft of the penis.

Case 2. A 37-year-old HIV-negative man presented with a genital ulcer and lymphadenopathy of 2 days' duration. He had multiple unprotected casual partners in sex parties 3 days before. Three consecutive dark-field exams were negative. Five days later the ulcer persisted like a chancre and inguinal buboes were more evident. LGV was detected from the ulcer while syphilis and herpes were negative by PCR. The clinical manifestations resolved quickly after completion of treatment with doxycycline as above.

Cases with non-rectal lesions would be expected as LGV spreads. When present, the most common presentation of the primary lesion in the genitalia is a nonindurated herpetiform evanescent ulcer. Clinicians must be vigilant to the evolving faces of LGV, be prepared to recognise the full manifestations of this disease and consider LGV in the differential diagnosis of genital chancre.

\section{P2.129 PREVALENCE OF MALARIA IN HIV PATIENTS UNDER COTRIMOXAZOLE, CASE OF KINDU, MANIEMA, D.R.CONGO}

doi:10.1136/sextrans-2013-051184.0393

'A M Bulabula, ${ }^{2} \mathrm{~N}$ A Bulabula, ${ }^{3} \mathrm{M}$ Mbo, ${ }^{3} \mathrm{~T}$ C Kayembe. ${ }^{1}$ Universite De Kindu, Kindu, Congo; 2 Provincial General Hospital of Bukavu, Bukavu, Congo; ${ }^{3}$ Universite de Kisangani, Kisangani, Congo

Background Malaria and HIV/AIDS are common in sub-Saharan Africa, their association aggravates the evolution of one another condition. HIV patients under cotrimoxazole have been reported to present less malaria cases. This study aims to determine the prevalence of malaria among HIV patients under Cotrimoxazole in Kindu.

Methods A sample of 1533 subjects, including 511 people living with HIV (PLHIV) attending the Voluntary Testing Centre (VCT) of the General Hospital of Kindu and 1022 controls was compiled. Thick and thin blood smears stained with Giemsa were used to search for asexual forms and determination of parasite species. The CD4 + lymphocytes count, in each patient was performed on FACS Count. We did a distribution of HIV patients in three groups according to the $\mathrm{CD} 4+$ range: $350 \mathrm{CD} 4+/ \mathrm{mm}^{3}$. Demographic data were recorded. The statistical analysis was performed on the software Epi Info 3.5.

Results 511 PLHIV 33.3\% (95\% CI, 31 -35.8) and 1022 controls $66.7 \%$ (95\% CI, 62.2 to 69$)$ are included in the sample. Females were dominant with $60.7 \%$ (95\% CI, 58.2 to 63.2 ). The average age was $34.6 \pm 12.6$ years. The overall prevalence of parasitaemia $17.5 \%$ (268/1533, 95\% CI: 15.6 to 19.6). Parasitaemia in PLHIV $13.7 \%$ $(70 / 511)$ against $19.4 \%(198 / 1022)$ in controls $(\mathrm{P}<0.05)$. Parasitaemia in PLHIV related to CD4 + was $16.4 \%(11 / 67)$ in the 350 CD4 $+/ \mathrm{mm}^{3}(\mathrm{P}<0.05)$

Conclusions Cotrimoxazole appears to protect PLHIV against malaria but there was not much difference with the controls.

\section{P2.130 PENILE MONDOR'S DISEASE: AN UNDERDIAGNOSED AND UNDER-REPORTED BENIGN CONDITION}

doi:10.1136/sextrans-2013-051184.0394

\section{B Kumar. Silver Oaks Multi Specialty Hospital, Mohali-Punjab, India}

Bhushan Kumar, Abir Saraswat, BD Radotra, Tarun Narang Background Mondor's disease of the penis is usually a benign, self-limiting process of acute onset; associated with considerable psychological stress and sexual disharmony. Although it is not so uncommon, there have been few large studies.

Methods A retrospective review of 37 patients with penile Mondor's disease over a period of 2002-2010 was done. Detailed history and clinical findings were recorded. Histopathological examination and staining with CD31 and CD34 monoclonal antibodies was also done in 17 patients. Patients were followed up to see the response to treatment and recurrences. 
The age of these patients ranged from $20-44$ years. The patients presented with cord-like thickening on penis within 24-48 hours after a prolonged sexual act with or without an intercourse. Seventeen patients had history of one or more episodes of STDs at presentation or in the past. Histopathological specimens showed prominent vessels with plump endothelial cells and thickened blood vessel walls. Occasional vessel showed complete occlusion of its lumen. Doppler US done in 10 patients showed dorsal vein thrombosis without the flow signals in this area. Patients were treated with counselling, abstinence and paracetamol. Thirty patients had resolution of the swelling by 6-8 weeks with treatment only two patient required surgery. No recurrence or erectile dysfunction was noted in any of the patients in the follow up from 2 to 8 years.

Conclusions Penile Mondor's disease has a favourable evolution and functional prognosis; although various etiologies have been proposed; trauma caused by sexual intercourse or masturbation is the main etiologic factor. Doppler US is a non invasive diagnostic modality helpful in both diagnosis and follow-up, however further analysis of Doppler US findings in a larger number of cases needs to be done to elucidate the hemodynamic changes in this not so rare entity.

\section{P2.131 A CLINICO-AETIOLOGICAL AND ULTRASONOGRAPHIC STUDY OF PEYRONIE'S DISEASE}

doi:10.1136/sextrans-2013-051184.0395

B Kumar. Silver Oaks Multi Specialty Hospital, Mohali- Punjab, India

Bhushan Kumar, Murlidhar, Tarun Narang

Background Peyronie's disease (PD) is caused by progressive fibrotic scarring of the tunica albuginea resulting in curvature or other deformities of the erect penis. PD is a psychologically and physically devastating disorder that manifests in middle-aged men. Although long recognised as an important clinical entity of the male genitalia, the aetiology of this disease is poorly understood.

Methods in this retrospective analysis we studied the epidemiology and clinical presentation of PD cases presenting to the dermatology out patient during Jan2001 - Dec2010. Diagnosis of PD was based on medical and sexual history, physical examination and imaging examinations: ultrasound, and colour Doppler ultrasound. Results Fifty-six men with PD presented during the period of ten years. Their ages ranged from 23 to 70 years. Most of them presented during the early phase of the disease. Most common presenting complaint was penile curvature in $80.95 \%$ followed by pain on erection in $66.66 \%$. History of penile trauma was revealed by $9.52 \%$ patients. Hypercholesterolemia (60\%), hypertension (33.3\%) and asymptomatic hyperuricemia $(28.34 \%)$ were the most common risk factors.32 patients with $\mathrm{PD}$ were studied by ultrasonography. Ultrasonogram was more accurate than clinical assessment in delineating the extent of lesions. In one-third of the patients, sonography demonstrated the plaques to be more extensive than had been detected by clinical examination.

Conclusions PD is a fibrotic wound-healing disorder involving the tunica albuginea of the corpora cavernosa leading to a variety of deformities of the genitalia which are associated with significant sexual dysfunction. The clinical symptoms and signs in our study were, in general, similar to those found in the previous studies. Higher incidence of hypertension and hypercholesterolemia in patients with PD may also be to an extent due to patients being in an older age group. However, it is difficult to explain asymptomatic hyperuricemia.

\section{P2.132 PHARYNGEAL AND CONJUNCTIVAL CHLAMYDIA TRACHOMATIS INFECTIONS: CHICKEN OR EGG?}

doi:10.1136/sextrans-2013-051184.0396

G Haidari, J A White. Guys \& St Thomas' NHS Foundation Trust, London, UK
Background Chlamydial inclusion conjunctivitis caused by genital serovars of Chlamydia trachomatis (CT) is well-recognised, and usually thought to result from auto-inoculation from genital CT infection or direct sexual contact. In this case series we review nine patients with co-existing pharyngeal and conjunctival CT infection and hypothesise on the relationship between the two conditions.

Methods Retrospective analysis of 9 patients with pharyngeal and conjunctival CT infection. Diagnoses were made based on clinical findings and the detection of CT RNA by the Aptima Combo2 assay (Gen-Probe).

Results We found 9 cases of coexisting pharyngeal and conjunctival infection in men: 8 were men who have sex with men (MSM) and 1 was heterosexual. All but one MSM had participated in both receptive and insertive oral sex, with the final patient reporting insertive oral sex and rimming.

All patients were symptomatic with unilateral conjunctivitis; one had symptoms bilaterally. Four of the 9 patients had a normal anogenital examination, and only 1 patient had a sore throat. Six of nine patients also had rectal CT infection, with 1 equivocal rectal CT result. Only two patients had co-existing urethral CT infection. Two patients had solely pharyngeal CT, including the heterosexual man.

Conclusions Whilst traditionally thought to be a result of autoinoculation from genital CT infection, we speculate that pharyngeal CT infection might be a more common source, or even a sequela, of CT conjunctivitis - at least in MSM. Alternatively, CT detected in the throat might be secondary to drainage of lacrimal fluid from a CT-infected eye. As CT conjunctivitis might be treated in isolation without comprehensive CT screening, or at most, with genital CT screening alone, we feel pharyngeal CT testing is indicated in all patients with CT conjunctivitis. The role of the nasolacrimal duct as a potential two-way conduit of infection requires further investigation.

\section{P2.133 PLASMA CELL BALANITIS (PCB). A CLINICO-PATHOLOGIC STUDY OF 132 CASES}

doi:10.1136/sextrans-2013-051184.0397

B Kumar. Silver Oaks Multi Specialty Hospital, Mohali-Punjab, India

Bhushan Kumar, Tarun Narang, B.D. Radotra

Background PCB also knows as Zoon balanitis, is a benign asymptomatic but chronic and erosive inflammatory condition of glans penis and prepuce that generally affects uncircumcised men in later years. Clinical presentation involves usually a single, shiny, well defined reddish patch. Disease may persist for years which adversely affects the quality of life of the individuals.

Methods 132 patients with a clinical diagnosis of PCB were studied between 2001-2010. Biopsy was done in patients who agreed for the procedure. Patients who did not agree for circumcision were prescribed fluticasone cream or tacrolimus $0.1 \%$ and were followed up. Results The age of the patients ranged from 24-70 years. Majority of patients had symptoms for more than 6 months. Lesions involved prepuce and glans in majority of patients; $82(62.12 \%)$, prepuce only in $24(18.18 \%)$ and glans only in $26(19.69 \%)$. Tissue for histopathology was available in 115 patients. Histologically, epidermal edoema, a dense upper dermal band of chronic inflammatory cells, including many plasma cells, dilated capillaries, extravasated red blood cells and hemosiderin deposition, were seen. In all the 115 cases PCB was successfully treated by circumcision. Remaining patients were treated with fluticasone or tacrolimus $0.1 \%$. The period of follow up ranged from $2-7$ years. Circumcised patients did not develop any recurrences. However the patients treated with fluticasone and tacrolimus had a course of relapses and remissions. Only one patient developed erythroplasia of Query at during the follow up. 